\title{
ELETRODOS QUIMICAMENTE MODIFICADOS APLICADOS À ELETROANÁLISE: UMA BREVE ABORDAGEM
}

\author{
Maria de Fátima Brito Souza \\ Departamento de Engenharia de Materiais - Faculdade de Engenharia Mecânica - Universidade Estadual de Campinas - CP 6122 \\ - 13083-970 - Campinas - SP
}

Recebido em 14/2/96; aceito em 8/8/96

\begin{abstract}
CHEMICALLY MODIFIED ELECTRODES APPLYED TO ELECTROANALYSIS: A BRIEF PRESENTATION. Chemically modified electrodes (CMEs) have been subject of considerable attention since its inception about 23 years ago. CMEs result of a deliberate immobilization of a modifier agent onto the electrode surface obtained through chemical reactions, chemisorption, composite formation or polymer coating. This immobilization seeks transfer the physicochemical properties of the modifier to the electrode surface and thus to dictate and control the behavior of the electrode/solution interface. In recent years the interest in CMEs has increased particularly to enhance the sensitivity and/or the selectivity of electroanalytical techniques. In general higher sensitivity and/or selectivity may be achieved by exploiting one or more of the following phenomena: electrocatalysis, preconcentration and interferents exclusion. This paper deals with the application of CMEs in electroanalysis, including a brief presentation of the more general procedures that have been employed for the modification of electrode surfaces.
\end{abstract}

Keywords: chemically modified electrode; electroanalysis.

\section{INTRODUÇÃO}

O termo eletrodo quimicamente modificado (EQM) foi introduzido no jargão eletroquímico por Murray e col. ${ }^{1}$ em 1975 para designar eletrodos com espécies quimicamente ativas, deliberadamente imobilizadas em suas superfícies, com o objetivo de pré-estabelecer e controlar a natureza físico-química da interface eletrodo/solução. A modificação deliberada da superfície do eletrodo sendo uma forma de impor e controlar sua reatividade e/ou seletividade, possibilita o desenvolvimento de eletrodos para vários propósitos e aplicações.

Os primeiros trabalhos envolvendo a preparação de eletrodos com superfícies modificadas surgiram no início da década de 70. Até então só eram utilizados eletrodos de materiais ditos "inertes" tais como carbono, ouro, platina e mercúrio.

O primeiro exemplo de uma modificação deliberada da superfície de um eletrodo foi o trabalho de Lane e Hubbard ${ }^{2}$. Estes pesquisadores adsorveram na superfície de eletrodos de platina várias olefinas funcionalizadas, explorando a propensão de grupos alceno quimisorverem-se sobre este metal. Neste trabalho pioneiro foram feitas observações importantes. Por exemplo, foi verificada a capacidade do ácido 3-alil-salicílico coordenar ferro seletivamente, em função do potencial aplicado. Este fato foi a primeira indicação da utilidade analítica de EQMs, pois demonstrou a capacidade de um grupo imobilizado complexar um íon metálico, e também a possibilidade de se direcionar a coordenação através da escolha do potencial aplicado.

Em $1975^{3}$ foi publicado um trabalho descrevendo a construção de um eletrodo quiral para eletrosíntese de álcoois a partir da redução de cetonas. Aminoácidos opticamente ativos foram covalentemente ligados à superfície de um eletrodo de carbono vítreo, via grupos carboxila. Também em 1975 surgiu o agora clássico artigo de Murray ${ }^{1}$ descrevendo a modificação química da superfície de eletrodos de $\mathrm{SnO}_{2}$ via organosilanos.

A partir destes trabalhos pioneiros, a preparação e utilização de EQMs têm se expandido de forma bastante acentuada, como pode ser inferido do expressivo número de artigos publicados sobre este tema nos últimos anos. Além da eletroanálise, que tem se destacado como uma das áreas mais ativas neste campo, os EQMs têm sido utilizados para fins outros que não analíticos, incluindo estudos básicos de eletrocatálise, de cinética de transferência de elétrons, de permeação de membranas, síntese eletroorgânica e fotoeletroquímica.

Neste artigo são descritas as formas de aplicação eletroanalíticas e os métodos de preparação mais comuns de EQMs. Informações mais profundas e detalhadas podem ser encontradas em excelentes artigos de revisão disponíveis na literatura ${ }^{4-10}$

\section{ELETROANÁLISE}

A grande maioria das aplicações analíticas de EQMs envolve técnicas voltamétricas ou amperométricas. Entretanto também são descritos na literatura alguns casos de aplicação de EQMs como eletrodos íon seletivos. No contexto deste artigo serão considerados apenas as técnicas eletrolíticas.

Do ponto de vista analítico a sensibilidade e/ou a seletividade de uma determinação deve aumentar com a utilização de um EQM para que seu uso seja justificado. Isto pode ser obtido por meio de um ou mais dos seguintes fenômenos: préconcentração, eletrocatálise e exclusão de interferentes. Em geral a obtenção de sensibilidades mais altas ocorre por préconcentração da espécie de interesse ou por eletrocatálise, sendo que a aplicação de qualquer destes princípios resulta em inerente aumento da seletividade. Ganhos adicionais neste item podem ser alcançados pela utilização de recobrimento com membranas poliméricas que bloqueiam o acesso de espécies interferentes à superfície do eletrodo.

\subsection{Eletrocatálise}

Como em qualquer processo catalítico o objetivo da eletrocatálise é reduzir a energia de ativação da reação, no caso uma reação envolvendo transferência de elétrons. A oxidação ou redução de um substrato que apresenta uma cinética de transferência de elétrons lenta, à superfície do eletrodo, é mediada por um sistema redox que pode trocar elétrons mais rapidamente com o eletrodo e o substrato nesta ordem (processo EC - eletroquímico-químico) ou na ordem inversa (processo CE químico-eletroquímico), reduzindo o sobrepotencial de ativação. O sobrepotencial de ativação é o potencial adicional a $\mathrm{E}^{\mathrm{o}}$, 
necessário para vencer a barreira da energia de ativação de uma dada eletrólise em um dado eletrodo, e manter a reação a uma dada velocidade. A redução do sobrepotencial de ativação aumenta a seletividade da medida porque permite a aplicação de potenciais de operação mais baixos, e a especificidade é inversamente relacionada à magnitude do potencial aplicado. Isto porque assim fica reduzida, ou mesmo eliminada, a possibilidade de que os potenciais de eletrólise de outras espécies presentes sejam atingidos.

Os mediadores redox podem ser espécies orgânicas (quinonas, tetratiofulvaleno; tetracianoquinodimetano, etc), compostos organometálicos (ferrocenos, ftalocianinas, óxidos de rutênio, metaloporfirinas, etc) ou enzimas. Estas espécies atuam em solução ou no caso de EQMs, imobilizadas à superfície do eletrodo, podendo ser utilizado mais de um mediador. A figura 1 ilustra esquematicamente o princípio da ação de um mediador imobilizado à superfície de um EQM, em um processo de (a) redução ou (b) oxidação de um substrato. Exemplos na tabela 1.

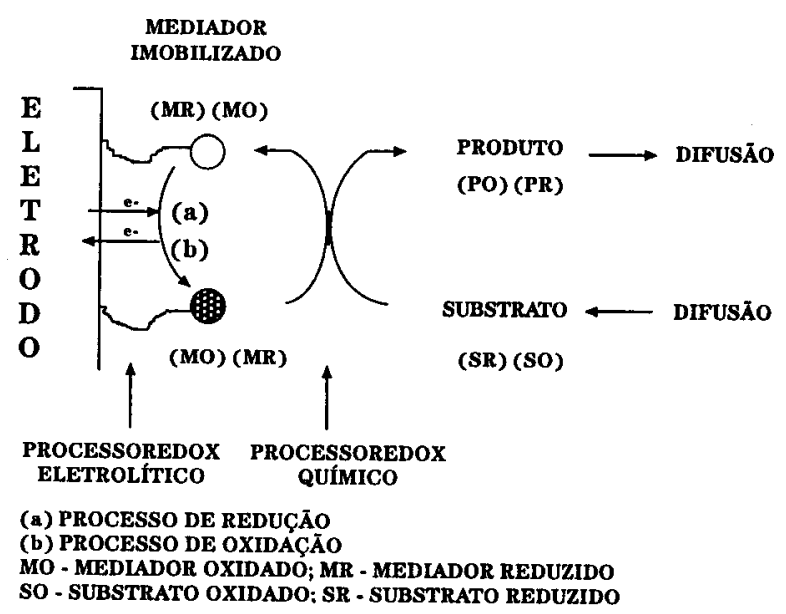

Figura 1. Ilustração esquemática do princípio de atuacão de um mediador redox imobilizado à superfície de um EQM usado para eletrocatálise.

\subsection{Pré-Concentração}

A sensibilidade das técnicas voltamétricas e amperométricas é diretamente relacionada à razão corrente faradaica (if)/corrente capacitiva (ic). Os métodos que incluem uma etapa inicial de pré-concentração do analito, possibilitam um aumento na componente faradaica da corrente e portanto elevam a sensibilidade da técnica voltamétrica utilizada. Em geral a concentração da espécie acumulada é de 10 a 1000 vezes maior na superfície do eletrodo do que originalmente em solução.

A forma convencional de pré-concentração, principalmente para íons metálicos, é a eletrodeposição, mas sua aplicação a amostras reais é muitas vezes limitada, ou pela presença de interferentes que são eletrodepositados juntamente com a espécie alvo, ou por ser o potencial de redução do íon de interesse muito negativo.

Em um EQM o fenômeno de pré-concentração envolve uma interação química (complexação, troca iônica, ligação covalente) entre a espécie a ser pré-concentrada e um agente modificador imobilizado à superfície do eletrodo. Este agente modificador, apresenta sob condições específicas, envolvendo ou não a aplicação de potencial, uma particular afinidade pela espécie alvo, o que confere seletividade à medida voltamétrica. Alternativamente a pré-concentração pode ser decorrência de um fenômeno de partição. É o que ocorre com certas espécies orgânicas hifrofóbicas que sofrem um processo de acumulação extrativa em eletrodos de pasta de carbono. Por ser a pasta de carbono uma mistura de carbono em pó com um líquido orgânico aglutinante (Nujol, Silicone, Vaselina, etc.) existe a possibilidade de algum composto orgânico eletroativo ser extraído pela pasta, devido à sua solubilidade na fase orgânica. Ainda uma outra forma de pré-concentração é possível, através da incorporação de uma espécie aniônica em um polímero condutor na forma oxidada. Este processo, conhecido como dopagem, é induzido eletroquimicamente. A tabela 2 ilustra a utilização de EQMs desenvolvidos para aplicação em determinações voltamétricas que envolvem uma etapa de pré-concentração não-eletrolítica.

\subsection{Exclusão de Interferentes}

A ação de interferentes, inibindo ou mascarando a resposta eletroquímica da espécie de interesse, pode ser devida, em geral, a um ou mais dos seguintes fenômenos:

a) passivação - adsorção do interferente (espécies tensoativas ou macromoléculas) à superfície do eletrodo o que impede o acesso da espécie de interesse, ou reduz a velocidade de troca de elétrons, por ex. em fluídos biológicos;

b) competição do interferente com a espécie de interesse pelos sítios ligantes existentes à superfície do eletrodo;

c) eletrólise do interferente, pré-concentrado à superfície do eletrodo ou em solução, em valor de potencial próximo ao potencial de eletrólise da espécie de interesse.

Uma maneira de promover exclusão, por meios físicos, é recobrir a superfície do eletrodo com uma membrana polimérica, que possa, com base na carga e/ou tamanho bloquear o acesso do interferente, sendo permeável à espécie de interesse.

Tabela 1. Exemplos de determinações voltamétricas e amperométricas, envolvendo eletrodos modificados com mediadores redox.

\begin{tabular}{|c|c|c|c|c|}
\hline $\begin{array}{c}\text { ELETRODO } \\
\text { BASE }\end{array}$ & $\begin{array}{l}\text { MÉTODO DE } \\
\text { MODIFICAÇÃO }\end{array}$ & $\begin{array}{c}\text { ESPÉCIE } \\
\text { DETERMINADA }\end{array}$ & $\begin{array}{l}\text { LIMITE DE } \\
\text { DETECÇÃO }\end{array}$ & REFERÊNCIA \\
\hline $\begin{array}{c}\text { grafite } \\
\text { espectroscópico }\end{array}$ & $\begin{array}{l}\text { imobilizaçao } \\
\text { covalente de } \\
\text { glicoseoxidase }\end{array}$ & glicose & $1 \times 10^{-6} \mathrm{M}$ & 11 \\
\hline $\begin{array}{c}\text { eletrodo de carbono } \\
\text { impresso sobre } \\
\text { PVC }\end{array}$ & $\begin{array}{l}\text { formação de } \\
\text { compósito (tinta } \\
\text { de carbono + Meldola's } \\
\text { Blue) }\end{array}$ & $\mathrm{NADH}$ & $2,5 \times 10^{-6} \mathrm{M}$ & 12 \\
\hline grafite-epóxi & $\begin{array}{l}\text { formação de } \\
\text { compósito com } \\
\text { tirosinase }\end{array}$ & $\begin{array}{l}\text { fenol e } \\
\text { catecol }\end{array}$ & $\begin{array}{c}0,04 \times 10^{-6} \mathrm{M} \\
1 \times 10^{-6} \mathrm{M}\end{array}$ & 13 \\
\hline
\end{tabular}

${ }^{a}$ Vide Item 3.2 
Tabela 2. Determinações voltamétricas com EQMs envolvendo etapa de acumulação.

\begin{tabular}{|c|c|c|c|c|c|}
\hline $\begin{array}{l}\text { ELETRODO } \\
\text { BASE }\end{array}$ & $\begin{array}{l}\text { MÉTODO DE } \\
\text { MODIFICAÇÃO }\end{array}$ & $\begin{array}{l}\text { ESPÉCIE } \\
\text { DETERMINADA }\end{array}$ & $\begin{array}{l}\text { LIMITE DE } \\
\text { DETECÇÃO }\end{array}$ & $\begin{array}{l}\text { FORMA DE } \\
\text { ACUMULAÇÃO }\end{array}$ & REF. \\
\hline $\begin{array}{l}\text { pasta de } \\
\text { carbono }\end{array}$ & $\begin{array}{l}\text { formação de } \\
\text { compósito com } \\
\text { sílica funcionalizada }\end{array}$ & $\mathrm{Hg}(\mathrm{II})$ & $\begin{array}{l}6 \times 10^{-7 \mathrm{M}} \\
(5 \mathrm{~min})\end{array}$ & complexação & 14 \\
\hline $\begin{array}{l}\text { pasta de } \\
\text { carbono }\end{array}$ & $\begin{array}{l}\text { formação de } \\
\text { compósito com } \\
\text { sais inorgânicos } \\
\left(\mathrm{PbSO}_{4}\right)\end{array}$ & $\mathrm{C}_{2} \mathrm{O}_{4}{ }^{2-}$ & $\begin{array}{l}3 \times 10^{-6} \mathrm{M} \\
(5 \mathrm{~min})\end{array}$ & $\begin{array}{l}\text { troca } \\
\text { iônica }\end{array}$ & 15 \\
\hline $\begin{array}{l}\text { carbono } \\
\text { vítreo }\end{array}$ & $\begin{array}{l}\text { recobrimento } \\
\text { com } \mathrm{PVP}^{\mathrm{a}}\end{array}$ & $\mathrm{Au}(\mathrm{III})$ & $\begin{array}{c}5 \times 10^{-8} \mathrm{M} \\
(60 \mathrm{~s})\end{array}$ & $\begin{array}{l}\text { troca } \\
\text { iônica }\end{array}$ & 16 \\
\hline platina & $\begin{array}{l}\text { adsorção de } \\
\text { alilamina }\end{array}$ & $\begin{array}{l}\text { ferroceno- } \\
\text { carboxialdeído }\end{array}$ & $\begin{array}{l}10^{-7} \mathrm{M} \\
(5 \mathrm{~min})\end{array}$ & $\begin{array}{l}\text { ligação } \\
\text { covalente }\end{array}$ & 17 \\
\hline
\end{tabular}

aPVP: poli(4-vinilpiridina)

Tabela 3. Determinações analíticas envolvendo EQMs e exclusão de interferentes

\begin{tabular}{|c|c|c|c|c|}
\hline $\begin{array}{l}\text { ELETRODO } \\
\text { BASE }\end{array}$ & $\begin{array}{l}\text { MÉTODO DE } \\
\text { MODIFICAÇÃO }\end{array}$ & $\begin{array}{l}\text { ESPÉCIE } \\
\text { DETERMINADA }\end{array}$ & $\begin{array}{l}\text { LIMITE DE } \\
\text { DETECÇÃO }\end{array}$ & REFERÊNCIA \\
\hline $\begin{array}{l}\text { carbono } \\
\text { vítreo }\end{array}$ & $\begin{array}{l}\text { recobrimento com } \\
\text { filme de Nafion } \\
\text { contendo éter de coroa }\end{array}$ & $\mathrm{Hg}(\mathrm{II})$ & $\begin{array}{c}10^{-9} \mathrm{M} \\
(5 \mathrm{~min} \text { acumulação) }\end{array}$ & 18 \\
\hline ouro & $\begin{array}{l}\text { adsorção de ácido } \\
\omega \text {-mercapto carboxílico } \\
\text { (mono-camada auto- } \\
\text { arranjada) }\end{array}$ & dopamina & $2 \mathrm{mM}^{\mathrm{a}}$ & 19 \\
\hline platina & $\begin{array}{l}\text { recobrimento com } \\
\text { membrana de PVC }\end{array}$ & $\begin{array}{l}\text { atividade de G6PDH } \\
\text { via oxidação de NADH }\end{array}$ & $0,1 \mathrm{U} / \mathrm{ml}$ & 20 \\
\hline $\begin{array}{l}\text { carbono } \\
\text { vítreo }\end{array}$ & $\begin{array}{l}\text { duplo recobrimento: } \\
1^{\circ} \text { ) membrana de } \\
\text { acetato de celulose } \\
2^{\circ} \text { ) membrana contendo } \\
\text { enzima imobilizada }\end{array}$ & glicose & $1 \times 10^{-6} \mathrm{M}$ & 21 \\
\hline
\end{tabular}

(a) Menor concentração utilizada pelos autores

Assim apenas esta chega até a superfície do eletrodo. Filmes tais como acetato de celulose ou Nafion, têm sido utilizados para este fim. Nafion é um polieletrólito catiônico, e apresenta portanto a tendência de repelir espécies aniônicas enquanto permite a passagem de cátions. Por sua vez os filmes de acetato de celulose atuam com base no tamanho das espécies envolvidas. Outra maneira de excluir interferentes, também fundamentada em interações eletrostáticas, é através da formação de monocamadas auto-arranjadas, que sejam seletivas. A tabela 3 apresenta exemplos de EQMs projetados para promover exclusão de interferentes.

\section{PREPARAÇÃO DE EQMS}

A utilização de EQMs é uma área em franco desenvolvimento, e a cada dia novos métodos de preparação são descritos. Aqui será feita apenas uma rápida abordagem deste tópico, procurando-se dar uma visão bem geral dos métodos de preparação mais comuns.

\subsection{Substrato Base}

A escolha do material para o eletrodo base, cuja superfície sofrerá a modificação, é um aspecto muito importante da preparação de um EQM. Este substrato deve apresentar características eletroquímicas apropriadas e também ser adequado para o método de imobilização selecionado. Entre os materiais convencionais estão o ouro, platina, carbono vítreo, mercúrio na forma de filme e pasta de carbono. Carbono vítreo reticulado, fibras de carbono, material plástico condutor e vidros condutores, estão incluídos entre os substratos menos usuais .

\subsection{Métodos de Imobilização do Modificador}

\section{Adsorção}

Adsorção, ou mais apropriadamente quimisorção, foi o processo pioneiro e é a maneira mais simples de fixar um modificador ao substrato do eletrodo base. Consiste na dissolução 
do agente modificador em um solvente apropriado, e na exposição, em geral por imersão, do eletrodo à esta solução. Pode também envolver a disposição desta solução, por exemplo com o auxílio de uma micro-pipeta, sobre a superfície a ser modificada, com posterior evaporação do solvente. Embora os primeiros estudos envolvendo adsorção (Lane e Hubbard) tenham utilizado eletrodos de platina, a maioria dos trabalhos subsequentes com adsorção foram realizados com eletrodos de grafite e carbono vítreo. Eletrodos de carbono apresentam uma particular capacidade de quimisorver reagentes que possuam sistemas de elétrons $\pi$ extendidos, como por exemplo compostos orgânicos aromáticos. Esta adsorção é devida à forte interação entre o plano basal (Figura 2) do eletrodo de carbono e o sistema de elétrons $\pi$ da molécula aromática.
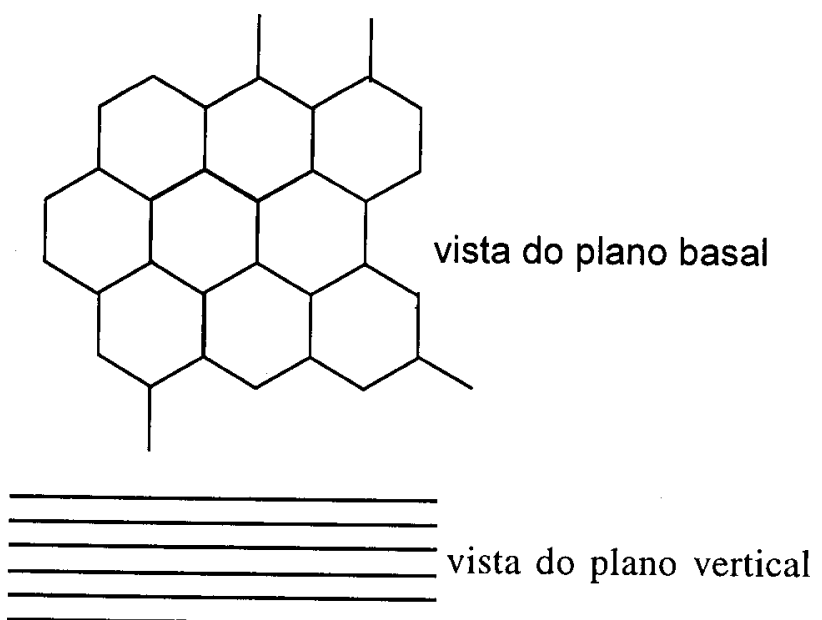

Figura 2. Ilustração esquemáticá dos planos basal (plano da folha) $e$ vertical (plano perpendicular à folha) de superfíwcies carbonáceas.

Embora simples, esta técnica apresenta a desvantagem de produzir EQMs com no máximo uma monocamada do modificador imobilizado, o que limita a faixa de resposta linear. Além disso, em tratando-se o fenômeno de adsorção de um processo de equilíbrio, inevitavelmente ocorrerá desorção do modificador para o meio, durante sua utilização, o que redunda em perda de reprodutibilidade, reduzindo portanto a vida útil do EQM assim preparado.

\section{Formação de compósitos}

Um compósito é uma mistura de componentes, portanto outra forma de preparar um EQM é simplesmente misturar o agente modificador com o substrato do eletrodo. Esta técnica é adequada para modificar eletrodos à base de carbono em pó (grafite, negro de carbono, etc.), tais como: eletrodos de pasta de carbono, de grafite-epóxi, "screen-printed"* e pastilhas.

\footnotetext{
Eletrodos "screen printed" (impressos) são obtidos a partir da deposição de uma suspensão de um material condutor sobre um suporte inerte (PVC, cerâmica de alumina, etc.). Esta deposição é efetuada ou por meio de um processo serigráfico, ou através de máquinas "screenprinters" semi-automáticas existentes no mercado, que utilizam "tintas condutoras" também comercialmente disponíveis. Neste caso, a tinta é derramada sobre o suporte na forma de um fino filete, à maneira de uma impressora "jato de tinta". No caso do processo serigráfico, a suspensão condutora passa através das partes permeáveis de uma espécie de extêncil, contendo o "gabarito" do eletrodo, premida por um puxador (rodo). Esta metodologia é particularmente apropriada para a preparação de eletrodos descartáveis. Embora, em função de parâmetros econômicos e eletroquímicos, as suspensões (tintas) de carbono sejam as mais utilizadas, suspensões de metais, como prata e ouro, são também utilizadas.
}

Quando possível é preferencialmente utilizada uma variante do método descrito anteriormente, que consiste em misturar o carbono em pó à uma solução adequada do agente modificador. Após a evaporação do solvente as partículas de carbono ficam recobertas pelo modificador, isto resulta numa distribuição mais homogênea deste último. Em ambos os casos também pode ocorrer perda gradual do modificador para à solução de medida, prejudicando a reprodutibilidade.

\section{Formação de Ligação Covalente}

O modificador pode também ser ligado covalentemente ao substrato do eletrodo. São empregadas por exemplo reações de silanização, envolvendo organosilanos e óxidos presentes à superfície do eletrodo. A maioria dos eletrodos metálicos, quando oxidados em meio ácido, são recobertos com uma camada fina de óxido, bastante reativa em relação a silanos. Portanto, um metal após ser oxidado, pode ser silanizado e posteriormente reagir com outra molécula, contendo o grupo funcional que se queira imobilizar. O silano atuará como um tipo de ponte para fixar um grupo funcional específico à superfície do eletrodo.

Superfícies de carbono, apresentam grupos óxidos funcionais tais como álcoois (fenóis), ácidos carboxílicos, cetonas (quinonas) e anidridos, resultantes da oxigenação de átomos de carbono do plano vertical, contendo ligações incompletas. Estas funções, cuja concentração pode ser aumentada através de reações de oxidação, são passíveis de derivatização. Portanto a modificação de superfícies de carbono, via ligação covalente do modificador, tem sido em grande parte baseada na manipulação da reatividade destes grupos funcionais, frente a reagentes como aminas, organosilanos, cloreto de tionila, e cloreto cianúrico, entre outros. Entretanto, também tem sido explorada a possibilidade de modificar covalentemente superfícies de carbono livres de óxidos. Nestes casos as reações envolvem os próprios átomos de carbono do plano vertical. Tratamentos como abrasão mecânica ou fratura sob atmosfera inerte, "decapagem" por plasma de argônio ou termólise sob vácuo, são capazes de gerar superfícies livres de óxidos, com grande reatividade frente a uma ampla variedade de reagentes.

Os eletrodos modificados via ligação covalente são mais estáveis em relação aos obtidos pelos demais métodos, entretanto são mais difíceis de preparar. Da mesma forma que a modificação por adsorção, esta metodologia também gera coberturas com no máximo uma monocamada imobilizada.

\section{Recobrimento com Membranas Poliméricas}

Outra técnica bastante atrativa para a preparação de eletrodos modificados é o recobrimento da superfície do eletrodo com filmes poliméricos, que devem ser condutores ou permeáveis ao eletrólito de suporte e à espécie de interesse. Ao contrário da modificação por adsorção ou por formação de ligação covalente, a modificação com membranas poliméricas permite a imobilização de muitas monocamadas (1 a 20000) da espécie ativa na superfície modificada, o que resulta na ampliação da resposta eletroquímica. Dependendo da aplicação pode ser escolhido um polímero eletroativo (se o objetivo é uma eletrocatálise), quimicamente ativo (propriedades ligante ou de troca iônica para préconcentração), ou inerte (apenas exclusão de interferentes). Os filmes eletroativos se subdividem em duas categorias principais, dependendo de como o centro redox é imobilizado: polímeros redox, se o centro redox é parte do esqueleto polimérico; e polímeros de troca iônica se o componente redox ativo é um contra-íon de um filme poli-iônico como polivinilpiridina ou Nafion.

A cobertura polimérica pode ser obtida a partir de soluções de polímeros pré-formados, ou através de polimerização in situ a partir de unidades monoméricas. Neste último caso, o recobrimento polimérico pode ser obtido via eletropolimerização ou por meio de métodos não eletroquímicos como por exemplo: 
polimerização ativada por plasma, foto-induzida por UV ou polimerização de organosilanos.

\section{CONCLUSÕES}

Desde os trabalhos pioneiros na década de 70, o tema EQMs, tem se revelado como uma interessante área de pesquisa e tem sido objeto de considerável desenvolvimento. Atualmente, é sem dúvida uma das mais intensas áreas da pesquisa em química analítica. Isto porque este tipo de eletrodo se apresenta como uma estratégia, na maioria das vezes simples, e eficiente, para o desenvolvimento de procedimentos e técnicas eletroanalíticas em que a sensibilidade e a seletividade são significativamente maiores que as obtidas com o emprego dos eletrodos convencionais. Além de propiciar a obtenção de sensores eletroanalíticos mais seletivos e sensíveis, outra característica importante dos EQMs é o fato de possibilitarem a utilização de reagentes imobilizados. A relevância de sistemas analíticos baseados em reagentes imobilizados reside na minimização da quantidade de reagente necessário para uma análise em particular, o que redunda em redução de custos. Em termos de perspectivas futuras, considerando que a busca por medidas mais sensíveis e seletivas é uma constante nos domínios da Química Analítica, a associação de EQMs com microeletrodos vem despontando como a mais promissora vertente da Eletroanálise, principalmente no campo de análises biológica e ambiental.

\section{REFERÊNCIAS}

1. Moses, P. R.;Wier, P; Murray, R. W.; Anal Chem. 1975, 47, 1882.

2. Lane, R. F.; Hubbard, A. T.; J. Phys. Chem. 1973, 77, 1401.
3. Watkins, B. F.; Behling, J. R.; Kariv, E.; Miller, L. L.; J. Am. Chem. Soc. 1975, 97, 3549.

4. Snell, K. D.; Keenan, A. G.; Chem. Soc. Rev. 1979, 8, 259.

5. Murray, R. W. In Electroanalytical Chemistry; Bard, A. J., Ed.; M. Dekker: New York, 1984, Vol. 13, p191.

6. Murray, R. W.;Ewing, A. G.; Durst, A. R.; Anal. Chem. 1987, 59, 279 A.

7. Abruña, H. D.; Coord. Chem. Rev. 1988, 86, 135.

8. Merz, A. Topics in Current Chemistry; Spring-Verlag: Berlin Heidelberg, 1990, Vol 152, p 49.

9. Arrigan, D. W. M.; Analyst 1994, 119, 1953.

10. Gilmartin, M. A. T.; Hart, J. P.; Analyst 1995, 120, 1029.

11. Sprules, S. D.; Hart, J. P.; Wring, S. A.; Pittson. R.; Analyst 1994, 119, 253.

12. Stoytcheva, M.; Nankov, N; Sharkova, V.; Anal. Chim. Acta 1995, 315, 101

13. Onnerfjorf, P.; Emneus, J.; Marko-Varga, G.; Gorton, L.; Ortega, F.; Dominiguez, E.; Biosens. Bioelectron. 1995, $10,607$.

14. Aleixo, L. M.; Sousa, M. F. B.; Godinho, O. E. S.; Oliveira Neto, G.; Gushikem, Y.; Moreira, J. C.; Anal. Chim. Acta 1993, 271, 143.

15. Cai, X.; Ogorevec, B.; Tavcar, G.; Kalcher. K.; Electroanalysis 1995, 7, 639.

16. Riley, P. J.; Wallace, G. G.; Electroanalysis 1991, 3, 191.

17. Price, J.; Baldwin, R. P.; Anal. Chem. 1980, 52, 1940.

18. Gao, Z.; Li, P; Zhao, Z; Microchem. J. 1991, 43, 121.

19. Malen, F.; Mandler, D.; Anal. Chem. 1993, 65, 37.

20. Treloar, P. H.;Christie, I. M.; Kane, J. W.; Crump, P.; Nkohkwo, A. T.; Vadgama, P. M.; Electroanalysis 1995, 7, 216.

21. Amine, A.; Kauffmann, J. -M.; Patriarche, G. J.; Talanta 1993, 40, 1157. 